\section{Effectiveness-Studie bestätigt Vorteil für Depot-Antipsychotikum}

\section{Gerade in besonders gefährdeten, schwierig zu behandelnden Patienten- gruppen zeigt sich der Nutzen der Depot-Gabe gegenüber der oralen Medikation.}

$\mathrm{D}$ ie Schizophrenie ist eine Krankheit, die mannigfaltig in das Leben der Betroffenen eingreifen kann, mitunter führt sie auch zu Delinquenz, wie man beispielsweise an der nicht geringen Zahl an forensischen Patienten mit Unterbringungsgrund Schizophrenie sehen kann. Gerade bei schizophrenen Patienten, die mit dem Justizsystem in Berührung kommen, ist die Adhärenz zur antipsychotischen Medikation ein besonderes Problem. Andererseits sollte aber gerade bei dieser Patientengruppe die Adhärenz gesichert sein. In randomisierten, (placebo-)kontrollierten klinischen Studien (randomized, controlled clinical trials, RCT) werden solche Patienten aufgrund der zahlreichen Ausschlusskriterien nicht eingeschlossen, was die Übertragbarkeit der so generierten Studienergebnisse erschwert. Ein probates Mittel, um solche Wissenslücken zu schließen, stellen EffectivenessStudien (im Gegensatz zu Efficacy-Studien) dar. In Effectiveness-Studien wird versucht, Patienten, die repräsentativ sind, in Studien einzuschließen, die möglichst das Routinevorgehen widerspiegeln.

Alphs et al. führten eine solche Effectiveness-Studie zum Vergleich einer Depot-Medikation mit oraler antipsychotischer Medikation durch. In die 15-monatige Multicenterstudie wurden schizophrene Patienten, die schon einmal inhaftiert waren, eingeschlossen. Die Patienten erhielten entweder monatlich eine Injektion von Paliperidon-Depot oder täglich eine orale Medikation mit entweder Aripiprazol, Haloperidol, Olanzapin, Paliperidon, Perphenazin, Quetiapin oder Risperidon. Die Aufteilung in diese beiden Gruppen wurde randomisiert vorgenommen, es wurde offen behandelt. Als primärer Endpunkt war die Zeit bis zum Behandlungsversagen definiert. Hierunter verstand man eine Festnahme/Inhaftierung, eine stationär-psychiatrische Aufnahme, ein Suizid, ein Behandlungsabbruch oder die Zugabe anderer Medikation wegen nicht ausreichender Wirksamkeit, Arzneimittelsicherheit oder Verträglichkeit oder eine vermehrte Inanspruchnahme psychiat-

rischer Interventionen, um eine Hospitalisierung zu verhindern. Es wurden 450 Patienten eingeschlossen.

Unter Paliperidon-Depot zeigte sich im Vergleich zur oralen Medikation eine signifikante längere mittlere Zeit bis zum Behandlungsversagen (HR: 1,43; $95 \%$-KI: 1,$09 ;-1,88, \mathrm{p}=0,011)$. Die Rate an Behandlungsversagen während der 15-monatigen Studienperiode betrug unter dem Depot $39,8 \%$, unter der oralen Antipsychotikagabe 53,7\%. Festnahme/Inhaftierung sowie stationär-psychiatrische Aufnahme stellten die Hauptgründe des Behandlungsversagens dar ( $21,2 \%$ vs. $29,4 \%$ bzw. $8,0 \%$ vs. $11,9 \%$ ) (Tab. 1).

\title{
Kommentar
}

Diese Untersuchung zeigt zum einen, dass mit einer Depot-Gabe tatsächlich ein zusätzlicher Nutzen für schizophrene Patienten im Vergleich zur oralen Medikation erzielt werden kann, und das eben nicht nur in einer hochselektierten Studienpopulation, sondern auch in einer schwierig zu behandelnden, besonders gefährdeten Patientengruppe. Solche Effectiveness-Studien sollten zu relevanten psychiatrischen Fragestellungen zukünftig vermehrt durchgeführt werden.

Prof. Dr. med. Hans-Peter Volz

Alphs L et al. Real-world outcomes of paliperidone palmitate compared to daily oral antipsychotic therapy in schizophrenia: a randomized, open-label, review board-blinded 15-month study. J Clin Psychiatry 2015; 76: 554-61

Tab. 1: Gründe für das erste Therapieversagen

\begin{tabular}{|c|c|c|}
\hline & $\begin{array}{l}\text { Paliperidon-Depot } \\
(n=226) n(\%)\end{array}$ & $\begin{array}{l}\text { Orale Antipsychotika }(\mathrm{n}=218) \\
\mathrm{n}(\%)\end{array}$ \\
\hline Festnahme/Inhaftierung & $48(21,2)$ & $64(29,4)$ \\
\hline Stationär-psychiatrische Aufnahme & $18(8,0)$ & $26(11,9)$ \\
\hline $\begin{array}{l}\text { Behandlungsabbruch wegen } \\
\text { - nicht ausreichender Sicherheit } \\
\text { oder Verträglichkeit } \\
\text { - nicht ausreichender Wirksamkeit }\end{array}$ & $15(6,6)$ & $\begin{array}{l}8(3,7) \\
9(4,1)\end{array}$ \\
\hline $\begin{array}{l}\text { Zugabe weiterer Medikamente } \\
\text { (wegen nicht ausreichende Wirk- } \\
\text { samkeit) }\end{array}$ & $5(2,2)$ & $6(2,8)$ \\
\hline $\begin{array}{l}\text { Vermehrte Inanspruchnahme psy- } \\
\text { chiatrischer Interventionen um } \\
\text { Hospitalisierung zu vermeiden }\end{array}$ & $3(1,3)$ & $4(1,8)$ \\
\hline Suizid & 0 & 0 \\
\hline Alle & $90(39,8)$ & $117(53,7)$ \\
\hline
\end{tabular}

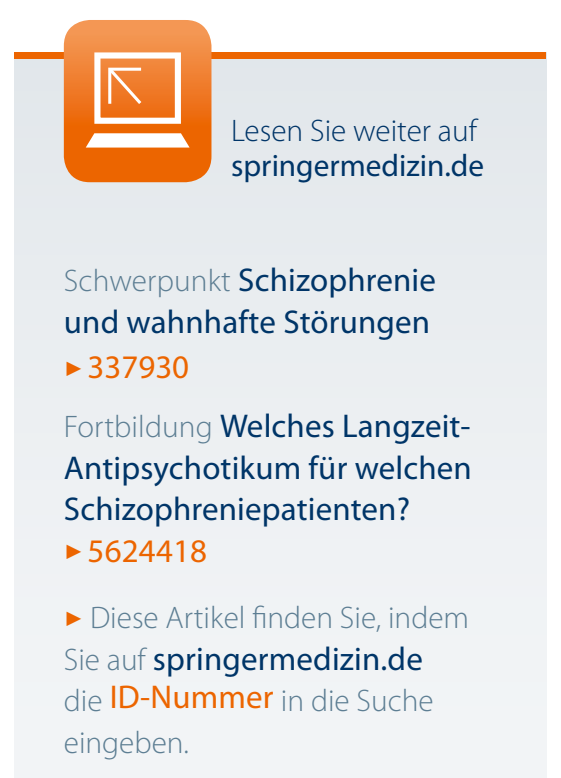

